\title{
Erratum to: Detection of IDH1 mutation in human gliomas: comparison of immunohistochemistry and sequencing
}

\author{
Shingo Takano - Wei Tian - Masahide Matsuda - Tetsuya Yamamoto • \\ Eiichi Ishikawa • Mika Kato Kaneko - Kentaro Yamazaki • Yukinari Kato • \\ Akira Matsumura
}

Published online: 1 April 2011

(C) The Japan Society of Brain Tumor Pathology 2011

\section{Erratum to: Brain Tumor Pathol \\ DOI 10.1007/s10014-011-0023-7}

The authors' affiliations appeared incorrectly in the article cited above. The correct authors' affiliations are as follows.

\section{S. Takano $(\bowtie) \cdot$ M. Matsuda · T. Yamamoto · E. Ishikawa ·} A. Matsumura

Department of Neurosurgery, Institute of Clinical Medicine, University of Tsukuba, 1-1-1 Tennoudai, Tsukuba, Ibaraki 305-8575, Japan

e-mail: shingo4@md.tsukuba.ac.jp

The online version of the original article can be found under doi:10.1007/s10014-011-0023-7.

S. Takano $(\bowtie) \cdot$ M. Matsuda - T. Yamamoto - E. Ishikawa ·

A. Matsumura

Department of Neurosurgery, Institute of Clinical Medicine, University of Tsukuba, 1-1-1 Tennoudai,

Tsukuba, Ibaraki 305-8575, Japan

e-mail: shingo4@md.tsukuba.ac.jp

W. Tian · M. K. Kaneko · Y. Kato

Molecular Tumor Marker Research Team,

The Oncology Research Center,

Advanced Molecular Epidemiology Research Institute,

Yamagata University Faculty of Medicine,

2-2-2 Iida-nishi, Yamagata 990-9585, Japan

K. Yamazaki

Department of Forensic Medicine,

Yamagata University Faculty of Medicine,

2-2-2 Iida-nishi, Yamagata 990-9585, Japan
W. Tian · M. K. Kaneko · Y. Kato

Molecular Tumor Marker Research Team, The Oncology Research Center, Advanced Molecular Epidemiology Research Institute, Yamagata University Faculty of Medicine, 2-2-2 Iida-nishi, Yamagata 990-9585, Japan

\section{K. Yamazaki}

Department of Forensic Medicine, Yamagata University Faculty of Medicine, 2-2-2 Iida-nishi, Yamagata 990-9585, Japan 